\title{
Standardized Study of Atorvastatin Possible Osteoarthritis Disease-Modifying Effect in Rats
}

\author{
Ali Gaballah ${ }^{1}$, Doaa Genedy ${ }^{1}$, Essam Ghayaty ${ }^{1}$, Amany Elhawwari ${ }^{1}$, and Ahlam Elmasry ${ }^{1}$ \\ ${ }^{1}$ Mansoura University Faculty of Medicine
}

November 12, 2020

\begin{abstract}
Background and purpose: Osteoarthritis (OA) is a chronic and progressive joint disorder characterized by structural damage to one or more joints. However, drugs that could cure or at least stop the progression of this disease are still given no satisfactory outcome. The purpose of this work is to study the potential OA disease-modifying effects of atorvastatin in an experimental model of osteoarthritis and the possible underlining mechanisms if any. Experimental Approach: Seventy-six adult male Sprague-Dawley rats (250-300gms) were used throughout this study. Forty rats were used to assess the effect of atorvastatin in surgically induced OA. While 36 rats were used to assess its anti-inflammatory effect in carrageenan-induced paw edema. In the model of OA; the degree of joint stiffness was assessed by measuring the angle of knee extension besides, the histopathological changes of the OA knee joints and measurement of serum Interleukin-1beta (IL-1 $\beta$ ), Matrix metalloproteinase-13 (MMP13), and reduced glutathione (GSH) concentration were biochemically assessed. In the carrageenan-induced paw edema, the paw thickness and pain threshold were assessed in different groups. Key Results: Atorvastatin was found to produce significant improvement of joint stiffness, the histopathological changes, a significant correction in the increased MMP13 and IL1- $\beta$, and the decreased GTH in OA rats. Also, atorvastatin showed a significant improvement in both paw thickness and pain threshold. Conclusion and Implications These results present atorvastatin as OA disease-modifying drug worse clinical trials.
\end{abstract}

Standardized Study of Atorvastatin Possible Osteoarthritis Disease-Modifying Effect in Rats. Short running title: Atorvastatin improves osteoarthritis in rats.

\section{Ali Gaballah, Doaa Genedy, Essam Ghayaty, Amany A. El-Hawwary, Ahlam Elmasry}

Ali M. Gaballah, Department of Clinical Pharmacology, Faculty of Medicine, Mansoura University, Egypt. 35516 .

E-mail; Profdr_ali@yahoo.com ; Tel: +20 1090649772

Doaa Genedy, Department of Clinical Pharmacology, Faculty of Medicine, Mansoura University, Egypt. 35516 .

E-mail; doctoraa91@gmail.com ; Tel: +20 1092196358

Essam Ghayaty, Department of Clinical Pharmacology, Faculty of Medicine, Mansoura University, Egypt. 35516 .

E-mail; EssamGhayaty@gmail.com; Tel: +20 1004712277

Amany A. El-Hawwary, Department of Histology, Faculty of Medicine, Mansoura University, Egypt. 35516.

E-mail; aelhawwari@yahoo.com ; Tel: +20 1122196932 
Ahlam Elmasry, Department of Clinical Pharmacology, Faculty of Medicine, Mansoura University, Egypt. 35516.

E-mail; ahlamielmasy@gmail.com; Tel: +201005608979

Corresponding author: Ahlam Elmasry

24 Gomhouria St., Department of Clinical Pharmacology, Faculty of Medicine, Mansoura, Egypt. 35516

Email: ahlamielmasy@gmail.com, Tel; 00201005608979

Availability of data :

The data that support the findings of this study are available from the corresponding author upon reasonable request.

\section{Acknowledgment:}

The authors gratefully acknowledge Dr. Basma Hamed, a veterinarian working in the MERC, Mansoura, for her help in the handling of the animals and experimenting. Also, the authors acknowledge Dr. Ahmed Mohamed Yousof; Assistant Lecturer of Community Medicine, Faculty of Medicine, Al- Alazhar University, for his help in the analysis of data using the SPSS program.

\section{Declarations of interest: none}

\section{Abstract}

Background and purpose : Osteoarthritis (OA) is a chronic and progressive joint disorder characterized by structural damage to one or more joints. However, drugs that could cure or at least stop the progression of this disease are still given no satisfactory outcome. The purpose of this work is to study the potential OA disease-modifying effects of atorvastatin in an experimental model of osteoarthritis and the possible underlining mechanisms if any. Experimental Approach: Seventy-six adult male Sprague-Dawley rats (250$300 \mathrm{gms}$ ) were used throughout this study. Forty rats were used to assess the effect of atorvastatin in surgically induced OA. While 36 rats were used to assess its anti-inflammatory effect in carrageenan-induced paw edema. In the model of OA; the degree of joint stiffness was assessed by measuring the angle of knee extension besides, the histopathological changes of the OA knee joints and measurement of serum Interleukin1beta (IL-1 $\beta$ ), Matrix metalloproteinase-13 (MMP13), and reduced glutathione (GSH) concentration were biochemically assessed. In the carrageenan-induced paw edema, the paw thickness and pain threshold were assessed in different groups.

Key Results: Atorvastatin was found to produce significant improvement of joint stiffness, the histopathological changes, a significant correction in the increased MMP13 and IL1- $\beta$, and the decreased GTH in OA rats. Also, atorvastatin showed a significant improvement in both paw thickness and pain threshold.

Conclusion and Implications: These results present atorvastatin as OA disease-modifying drug worse clinical trials.

Keywords: atorvastatin; carrageenan; IL1- $\beta \cdot$ MMP13; osteoarthritis; paw edema

\section{Introduction}

Osteoarthritis is a progressive joint disease affecting all joint structures. It is estimated to be the fourth leading cause of joint disability worldwide in addition to the high socioeconomic costs in all countries (Johnson and Hunter, 2014). Most available drug therapy can relieve symptoms of OA successfully but drugs that could cure or slow down the progress of the disease called disease-modifying osteoarthritic drugs (DMOAD) still do not produce a satisfactory outcome and considerable conflict and controversy about their effects and tolerability are present (Rovati et al., 2012). Therefore, the development of a new DMOAD is highly recommended. 
Statins, inhibitors of 3-Hydroxy-3-methylglutaryl coenzyme A (HMG-CoA) reductase, have been commonly used in cardiovascular diseases. In addition to the ability of statins to decrease cholesterol synthesis, statins have beneficial effects in various disease conditions, mediated through its cholesterol independent pleiotropic effects such as anti-inflammatory, antioxidant properties, and its attenuating tendency to affect a broad range of MMPs (Ghaisas et al., 2010). These properties make statins a possible candidate to be tried as DMOAD.

The present study aims to investigate the potential OA disease-modifying effect of atorvastatin and compares its effectiveness a previously used drug, glucosamine in an experimental model of OA and to provide a piece of direct experimental evidence for its possible underlying mechanism of ${ }^{*}$ action by assessing MMP3, IL1$\beta$, reduced glutathione in addition to the detection of its possible anti-nociceptive and anti-inflammatory effects.

\section{Materials and Methods}

\section{Drugs and chemicals}

Atorvastatin (tablets 10mg EIPICO), Glucosamine sulfate (capsules 500mg, EMA pharm), Indomethacin (capsules 25mg, Aspen pharma), Carrageenan raw material powder, Sigma Aldrich.

\section{Experimental animals}

Sixty-two adult male Sprague-Dawley rats (250-300 gm) obtained from Mansoura experimental Research Center (MERC) and were utilized in this study. They were put in similar optimum housing conditions with free access to food and water. Animals were kept in cages (4 rats/cage) at a room with a controlled temperature of $26^{\circ} \mathrm{C}$ and on a 12-h light-dark cycle. The institutional research board (IRB), Faculty of Medicine, Mansoura University animal ethics committee has approved all the experimental procedures under approval no (MS/17.08.76). Two experimental models were conducted: surgical induced OA and carrageenaninduced paw edema model.

\section{Surgical induction of OA}

Thirty-two rats were used. The surgical induction of OA was conducted on 24 rats according to (Janusz et al., 2002), while the remaining 8 rats served as the SHAM control group.

Experimental designThe rats were divided into 4 groups; the Sham non-OA group received saline; OA non-treated group received saline. Glucosamine treated OA group: received glucosamine sulfate solution at a dose of $250 \mathrm{mg} / \mathrm{kg} /$ day (Wen et al., 2010). Atorvastatin treated OA group: given atorvastatin solution $10 \mathrm{mg} / \mathrm{kg}$ daily (Pathak et al., 2015a). All drugs are given by oral gavage daily from $1^{\text {st }}$ day of surgery for six weeks.

\section{Joint stiffness assessment.}

After the scarification of the animals, the left knee of the animals was dissected, and the articular cartilage was left intact. The maximum extension angle of each knee was measured after the dissection with a zero degree indicates no stiffness increase value of the angle indicates limited joint movement and points to joint stiffness. The angle measured not the usual angle that is assumed to be, but it's a complementary angle (figure 1). After joint dissection, the joint was put on a paper and the maximum angle was measured by protractor (Rezende et al., 2006).

\section{Histopathological assessment}

The preparation of the knee specimen for the histopathological examination was done according to Schmitz et al. (Schmitz et al., 2010). Sections of five microns of tissue were cut then stained with hematoxylin and eosin $(\mathrm{H} \& \mathrm{E})$ for the examination of cartilage and subchondral bone. Semi-quantitative histological lesions grading was performed following the scoring system of (Khan et al., 2013).

\section{Biochemical assessment}


Blood was withdrawn from the heart of rats and was separated into 2 tubes; a dry tube for collection of sera to measure MMP13 and IL-1 $\beta$ and heparinized one for collection of erythrocyte lysate to measure GSH. Measurement of interleukin1- $\beta$ using rat Interleukin $1 \beta$ enzyme-linked immunosorbent assay (ELISA) kit Bioassay technology laboratory, Catalog No E0119Ra. Measurement of matrix metalloproteinase- 13 using rat MMP- 13 kit (Eiaab science com, Catalog No E0099r, China). Measurement of GSH by colorimetric method using a Bio-diagnostic kit (Egypt) (Beutler et al., 1963).

\section{Carrageenan induced paw edema}

Thirty rats were used for this model. Induction of paw edema was done by sub-plantar injection of carrageenan in the right hind limb of the 24 rats while 6 non injected rats serve as control normal. The animals were pretreated by oral gavage of the drugs, $1 \mathrm{~h}$ before carrageenan injection. Then, the rats received 0.1 $\mathrm{ml}$ of a $1 \%(\mathrm{w} / \mathrm{v})$ suspension of carrageenan in the sub-plantar surface of the right hind paw (Winter et al., 1962).

\section{Experimental design}

The control normal group received $0.5 \mathrm{ml}$ saline orally before sub-plantar injection of $0.1 \mathrm{ml}$ of saline. Control carrageenan-treated group rats pretreated with $0.5 \mathrm{ml}$ saline by gavage before sub-plantar injection of 0.1 $\mathrm{ml}$ of $1 \%$ carrageenan dissolved in saline (Winter et al., 1962). Indomethacin-pretreated group received an indomethacin solution at a dose of $10 \mathrm{mg} / \mathrm{kg}$, served as a standard drug group (Sadeghi et al., 2013). Glucosamine pretreated group received glucosamine sulfate solution by oral gavage at a dose of $250 \mathrm{mg} / \mathrm{kg}$ (Singh et al., 2007). The atorvastatin-pretreated group received atorvastatin at a dose of $10 \mathrm{mg} / \mathrm{kg}$ (Ghaisas et al., 2010).

\section{Recording of paw thickness:}

The paw thickness was recorded from the ventral to the dorsal surfaces using a dial thickness gauge flat contact point $0-10 \mathrm{~mm}$ tester leather measuring tool (weldingstop,china) at $1,2,3,4 \mathrm{~h}$, and $24 \mathrm{~h}$ after carrageenan injection (Solanki et al., 2015).

\section{Recording of pain threshold:}

Carrageenan-induced hyperalgesia was assessed using an analgesimeter (Ugo Basile, Comerio, Italy) which was composed of a cone-shaped paw-pressor with a rounded tip that was used to apply linear increasing force to test paw at the metacarpal level of right dorsal surface oh hind paw. The nociceptive threshold was taken as the point at which the rat vocalized or struggled vigorously, expressed as the force in grams (g). The nociceptive threshold $(\mathrm{g})$ was recorded at $1,2,3,4 \mathrm{~h}$, and $24 \mathrm{~h}$ after carrageenan injection (Marius et al., 2018).

\section{Statistical Analysis}

The results were statistically analyzed using the Statistical Package for Social Science (SPSS) program. The parametric results were expressed as Mean \pm SD. One-way analysis of variance (ANOVA) followed by post hoc Tukey's multiple comparisons was used for statistical analysis between groups. Kruskal Wallis test was used for comparison of means of more than two different groups of non-parametric data followed by posthoc Dunne's test, data were presented as median \& range (minimum-maximum). For all above mentioned statistical tests done, the threshold of significance is fixed at $5 \%$ level (p-value) or less.

1. Results

2. Model of OA:

3. Assessment of degree of joint stiffness by recording the maximum angle of knee extension in different groups :

Surgical induction of $\mathrm{OA}$ in rats produced a statistically significant increase in the maximum angle of knee extension compared to the control normal rats. Glucosamine and atorvastatin showed a statistically 
significant correction of the increased maximum angle of knee extension found in the non treated OA group. Atorvastatin treated OA group is significantly less than the glucosamine treated OA group, (Table 1).

\section{Effect of different drugs on histopathological changes in osteoarthritic rats:}

Histopathological examination of the control osteoarthritic rats showed loss of superficial layer, cell death, hypocellularity, the maximum loss of cellular layers and matrix, erosion and cavitation in the subchondral bone (figure 2) and showed a significant increase in the histopathological score as compared to control non-arthritic rats, (Table 1).

The histopathological examination of the glucosamine and atorvastatin treated OA groups showed improvement of osteoarthritic changes as shown in (figure 2), and histological score as compared to the non-treated OA group, but still significantly higher than the control normal

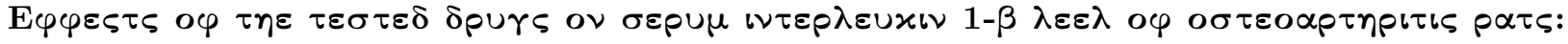

Surgical induction of OA in rats produced a significant rise in serum IL1- $\beta$ compared to the control normal rats. Glucosamine and atorvastatin treated OA groups showed a statistically significant reduction in the raised IL1- $\beta$ noticed in the non-treated OA group( Table1).

\section{Serum matrix metalloproteinase 13 levels in different groups:}

Osteoarthritic rats showed a significant increase in serum MMP13 compared to the control non-osteoarthritic rats. Glucosamine and atorvastatin treated OA groups showed a statistically significant correction of the increased MMP-13 found in the non-treated OA group. Atorvastatin reduction is significantly less than other treated groups, (Table1).

\section{Effect of tested drugs on GSH level in osteoarthritic rat groups:}

Surgical induction of OA in rats produced a statistically significant decrease in GSH compared to the control normal rats. Glucosamine and atorvastatin OA treated groups showed a statistically significant correction of the reduced GSH found in the non-treated OA group. Atorvastatin correction of GSH is significantly higher than glucosamine treated OA groups, (Table 1).

\section{Assessment of the anti-inflammatory and analgesic effects of tested drugs on an experimental model of inflammation}

\section{Effect on Paw edema}

Sub-plantar injection of carrageenan produced a significant increase in paw thickness at 1,2,3,4 and 24 hours as compared to control normal group (figure 3). Indomethacin, glucosamine, and atorvastatin pretreated groups showed a statistically significant reduction of increased paw thickness induced by carrageenan starting from $1^{\text {st }}$ hour then at $2,3,4$, and 24 hours compared to the carrageenan treated group (figure 3 ). Atorvastatin pretreated group showed a statistically more significant reduction of the increased paw thickness than glucosamine pretreated groups. However, the reduction of the increased paw thickness in glucosamine or atorvastatin pretreated group significantly less than that produced by indomethacin, (figure 3).

\section{Effect on nociceptive threshold}

Sub-plantar injection of carrageenan in rats produced a statistically significant decrease in the nociceptive threshold at 1, 2, 3, 4, and 24 hours as compared to the control normal group. Indomethacin, glucosamine, or atorvastatin pretreated groups showed a statistically significant improvement in the reduced nociceptive threshold. The improvement found in the indomethacin pretreated group is significantly more than the other tested drugs Atorvastatin pretreated group showed a more significant improvement of the decreased nociceptive threshold than glucosamine treated groups at all-time intervals, (figure 4)

\section{Discussion}

Effect of atorvastatin or glucosamine sulfate on osteoarthritic rats : 
The histopathological examination of non-treated OA rats showed several osteoarthritic changes that are following Bagi et al., 2015; Marino-Martinez et al., 2019. Surgically-induced models of OA largely mimic the human OA histopathologically (Kuyinu et al., 2016).

Glucosamine treated group showed improvement of histopathological changes that occurred in the nontreated OA group in agreement with Wen et al., 2010 and Waly et al., 2017. Atorvastatin treated group showed a significant improvement of the histopathological score with an improved microscopic picture of the examined knee joint specimens to be almost near normal. These results are in agreement with Pathak, Lingaraju, et al., 2015 and Gaballah et al., 2015.

A significant increase in the maximum angle of knee extension in the osteoarthritic group, as a sign of joint stiffness, is in agreement with previous studies (Choi et al., 2015; Phan et al., 2015). The joint stiffness that occurs in OA mainly results from a chronic inflammatory process that progresses and leads to fibrosis and then dysfunction of the joint (Kim et al., 2012). Glucosamine and atorvastatin treated groups showed significant correction of the joint stiffness found in the non-treated osteoarthritic group. This improvement of the joint stiffness is assumed to be due to their anti-inflammatory effect that reducing the joint fibrosis.

In the present study, surgical induction of OA was associated with a significant increase in IL-1 $\beta$ in agreement with previous studies by (Wei et al., 2018; Castrogiovanni et al., 2019). IL-1 $\beta$ alters the homeostatic balance of chondrocytes as it suppresses anabolic activity, and it decreases the expression of type II collagen and aggrecan and it inhibits glucuronosyltransferase which plays an important role in glycosaminoglycan biosynthesis. Also, it stimulates articular cartilage breakdown by increasing the expression of MMPs, chondrocyte apoptosis, and increasing the production of inflammatory mediators and reactive oxygen species (ROS)(Kapoor et al., 2011; Mobasheri and Batt, 2016).

The increase in IL-1 $\beta$ in OA rats was associated with a significant increase in serum level of MMP-13 following Ali et al., 2017; Castrogiovanni et al., 2019. Under normal conditions, there is a dynamic equilibrium between the synthesis and the degradation of the extracellular matrix component in osteoarthritic states, a disruption of matrix equilibrium leads to apoptosis of chondrocytes and cartilage degradation (Maldonado and Nam, 2013). MMPs are the most important proteinases responsible for extracellular matrix degradation. MMPs are activated by abnormal environmental insults, including high-magnitude mechanical stress, inflammatory mediators (Shiomi et al., 2010).

The increase of IL-1 $\beta$ following the induction of OA was significantly reduced by glucosamine. This finding was in line with Shahine \& Elhadidi, 2014. Glucosamine is assumed to decrease the serum level of IL-1 $\beta$ as it decreases interleukin 1-induced gene expression (Rovati et al., 2012). Glucosamine also limited the rise of MMP-13 produced by the induction of OA. This effect is in agreement with studies of (Rovati et al., 2012; Gibson et al., 2014).

Atorvastatin treated group in this study showed a significant reduction in the rise of IL- $\beta$ in agreement with Barsante et al., 2005; Simopoulou et al., 2010. This decrease in IL-1 $\beta$ level with atorvastatin is due to inhibition of production of isoprenoid derivatives that results in inhibition of $\mathrm{NF}-x \mathrm{~B}$ that regulates transcription of many inflammatory mediators including the IL-1 $\beta$ and mutagenic signaling pathway (Baker et al., 2011).

Administration of atorvastatin to OA rats showed a significant reduction in the rise of MMP-13. Simopoulou et al., 2010 suggested that atorvastatin may have possible chondroprotective effects, mainly by decreasing cartilage degradation protein MMP13. Pathak et al., 2015b noticed that atorvastatin significantly inhibited the IL-1 $\beta$-induced increased production of MMP-13 in an in-vitro OA model.

The effect of statins on MMPs may be due to inhibition of mevalonate synthesis which in turn plays an essential role in the regulation of several cellular mechanisms, including cytoskeletal dynamics and endocytic/exocytic transport. They are involved in MMP secretion, as well as transcription and synthesis of inflammatory cytokines and reactive oxygen species (Kavalipati et al., 2015).

Levels of reduced GSH in the erythrocyte lysate of osteoarthritic rats were reduced suggesting increased 
oxidative stress following Cifuentes et al., 2010; Regan et al., 2008. Increased ROS in OA is mainly associated with the reduction of the cartilage mass due to both inhibiting synthesis of cartilage matrix and inducing cartilage matrix breakdown (Henrotin et al., 2005). The glucosamine treated group in this study showed a significant elevation of the reduced GSH. Glucosamine was shown to possess antioxidant capacity (Katoh et al., 2017; Dai et al., 2018).

Atorvastatin treated group in this study showed a significant elevation of the decreased GSH. A similar antioxidant effect of atorvastatin has been reported by Pathak, Balaganur, et al., 2015 and Gaballah et al., 2015, who noticed that the GSH levels were restored to the normal by atorvastatin in an experimental model of osteoarthritis. The antioxidant effect of atorvastatin could be due to either increased biosynthesis of GSH or reduced oxidative stress. A recent study by (Hosseinzadeh et al., 2019) declared that atorvastatin enhanced the mRNA expression of antioxidant enzymes including glutathione peroxidase in cultured chondrocytes. Atorvastatin mitigated the production of ROS by endothelial cells by inhibiting NADPH oxidase activity via Rho-dependent mechanisms. Moreover, atorvastatin binds to erythrocyte membrane phospholipids or to lipoprotein fractions to prevent the diffusion of free radicals generated under oxidative stress (Bellosta et al., 2000).

Direct assessment of the anti-inflammatory and analgesic effects: It was found that sub-plantar injection of carrageenan produced a significant increase in paw thickness measured after 1,2,3,4, 24h. These results came in agreement with Sadeghi et al., 2014 and Antonisamy et al., 2017. The carrageenan-treated group also showed a significant decrease in the nociceptive threshold that was parallel with Kuedo et al., 2016; Marius et al., 2018. The indomethacin treated group showed a significant reduction of the increased paw thickness produced by carrageenan in agreement with Uzkeser et al., 2012 and Okhuarobo and Ozolua, 2017. It also showed a significant analgesic effect in agreement with Kuedo et al., 2016; Uzkeser et al., 2012. Indomethacin was used in the present study as a well-known analgesic anti-inflammatory standard drug to which the tested drugs are compared.

Glucosamine pretreated group showed a significant reduction in the increased paw edema. Setnikar et al., 1991 showed that glucosamine protected against the edema provoked by carrageenan, In contrast, Singh et al., 2007 noticed that glucosamine caused a non-significant decrease of paw edema after carrageenan injection. Glucosamine produced a significant correction of carrageenan-induced hyper nociceptive response in parallel to Wen et al., 2010. Antinociceptive effect of glucosamine may be due to its anti-inflammatory effect; suppressing the raised IL-1, ROS, and neutrophil functions.

Atorvastatin pretreated group produced a significant reduction of carrageenan-induced paw edema following Jaiswal and Sontakke, 2012. atorvastatin treated group showed significant inhibition of the hyper nociceptive response following Santodomingo-Garzon et al., 2006 and Kamel et al., 2016

Atorvastatin inhibition of carrageenan-induced rat paw edema may be due to inhibition of prostaglandins and other inflammatory mediators such as IFN- $\gamma$, TNF- $\alpha$, IL-1, and IL-6. Atorvastatin inhibits leukocyteendothelial adhesion, reduces the levels of inducible nitric oxide synthase and inhibits the production of monocyte chemotactic protein-1. These anti-inflammatory effects are due to the inhibition of NF-kB that regulates the transcription of many inflammatory mediators and mutagenic signaling pathways (Baker et al., 2011). Statins also activate anti-inflammatory transcription factors known as peroxisome proliferatoractivated receptors (PPARs) that interfere with NF-kB transcriptional activity (Cernuda-Morollón et al., 2002). Moreover, atorvastatin pretreatment showed correction of carrageenan-induced hyper nociception by inhibiting the production of proinflammatory mediators and deceased PGE2 sensitization action on nociceptors (Santodomingo-Garzon et al., 2006).

The pleiotropic effects of atorvastatin; including anti-inflammatory, analgesic, anti-catabolic, and antioxidant effects are assumed to have a beneficial role in the improvement of OA. The measurement of IL-1b and mmp-13 were performed to assess the anti-inflammatory and anticatabolic effects of atorvastatin. Besides, GSH assessment is used to detect the antioxidant effect of atorvastatin. Inflammation, oxidative stress are claimed to have a role in the catabolic state that occurs in OA. These pleiotropic effects are assumed to 
have a beneficial role in the improvement of OA. Also, it is reported that the expression of genes regulating cholesterol efflux is impaired in human OA chondrocytes, resulting in a toxic accumulation of lipid droplets in the chondrocyte that has a critical role in the development of OA (Tsezou et al., 2010). Atorvastatin has an anti-atheromatous effect that reduces structural deterioration of OA joints by improving the blood flow as the reduced blood flow in the small vessels in the subchondral bone may deteriorate the cartilage homeostasis and cause OA changes (Hoeven et al., 2013). Besides, atorvastatin is assumed to have an anabolic effect that is protective against cartilage damage (Karasawa, 2010). Atorvastatin may also inhibit osteoclasts activation by preventing mevalonate production, which leads to the loss of prenylation of small Ras and Rho GTPases and, consequently, disruption of downstream intracellular signaling pathways in osteoclasts (Hughes et al., 2007).

\section{Conclusion :}

The present study could present atorvastatin as a new useful potential DMOAD worse clinical trial for the treatment of OA. Accordingly, atorvastatin may be a promising DMOAD for treatment of OA especially in elderly patients suffering from hyperlipidemia, atherosclerosis.

Limitation of the studyOther markers which are assumed to be involved in the pathogenesis of OA have to be assessed. An assessment of other statins as a drug group has similar effects on OA like atorvastatin or not. Investigate the significance of atorvastatin cholesterol-lowering and anti-atheromatous action in correction subchondral bone ischemia. Also, an electron microscope examination of the affected joints may be of value to get a more precise evaluation.

\section{References}

A. Gaballa, N. Risk, A. Elhawary, E. Farrage, and E.G. (2015). Potential prophylactic effect of oral versus intraarticular atorvastatin in experimental model of osteoarthritis. Mansoura Med. J. 44 : 145-168.

Ali, S.M., Okda, A.A.K., Dessouky, I.S., Hewedy, W.A., Zahran, N.M., and Alamrani, B.A. (2017). 1 Carnitine ameliorates knee lesions in mono-iodoacetate induced osteoarthritis in rats . Alexandria J. Med.53 :61-66.

Antonisamy, P., Dhanasekaran, M., Kim, H.-R., Jo, S.-G., Agastian, P., and Kwon, K.-B. (2017). Antiinflammatory and analgesic activity of ononitol monohydrate isolated from Cassia tora L. in animal models. Saudi J. Biol. Sci. 24 : 1933-1938.

Bagi, C.M., Zakur, D.E., Berryman, E., Andresen, C.J., and Wilkie, D. (2015). Correlation between muCT imaging, histology and functional capacity of the osteoarthritic knee in the rat model of osteoarthritis. J. Transl. Med. 13 : 276.

Baker, J.F., Walsh, P., and Mulhall, K.J. (2011). Statins: a potential role in the management of osteoarthritis? Joint. Bone. Spine $78: 31-34$.

Barsante, M.M., Roffe, E., Yokoro, C.M., Tafuri, W.L., Souza, D.G., Pinho, V., et al. (2005). Antiinflammatory and analgesic effects of atorvastatin in a rat model of adjuvant-induced arthritis. Eur. J. Pharmacol. $516: 282-289$.

Bellosta, S., Ferri, N., Bernini, F., Paoletti, R., and Corsini, A. (2000). Non-lipid-related effects of statins. Ann. Med. 32 : 164-176.

Beutler, E., Duron, O., and Kelly, B.M. (1963). Improved method for the determination of blood glutathione. J. Lab. Clin. Med. $61: 882-888$.

Castrogiovanni, P., Rosa, M. Di, Ravalli, S., Castorina, A., Guglielmino, C., Imbesi, R., et al. (2019). Moderate Physical Activity as a Prevention Method for Knee Osteoarthritis and the Role of Synoviocytes as Biological Key. Int. J. Mol. Sci. 20 :. 
Cernuda-Morollón, E., Rodríguez-Pascual, F., Klatt, P., Lamas, S., and Pérez-Sala, D. (2002). PPAR Agonists Amplify iNOS Expression While Inhibiting NF- $x$ B: Implications for Mesangial Cell Activation by Cytokines. J. Am. Soc. Nephrol. 13 : 2223 LP - 2231.

Choi, J.-S., Shin, H.-S., Kim, K.Y., Ku, S.K., Choi, I.S., and Kim, J.W. (2015). Effect of Polycalcium, a mixture of Polycan and calcium lactate-gluconate in a 1:9 weight ratio, on rats with surgery-induced osteoarthritis. Exp. Ther. Med. $9: 1780-1790$.

Cifuentes, D.J., Rocha, L.G., Silva, L.A., Brito, A.C., Rueff-Barroso, C.R., Porto, L.C., et al. (2010). Decrease in oxidative stress and histological changes induced by physical exercise calibrated in rats with osteoarthritis induced by monosodium iodoacetate. Osteoarthr. Cartil. $18: 1088-1095$.

Dai, W., Qi, C., and Wang, S. (2018). Synergistic effect of glucosamine and vitamin E against experimental rheumatoid arthritis in neonatal rats. Biomed. Pharmacother. 105 : 835-840.

Dong, H., Sun, H., Magal, E., Ding, X., Kumar, G.N., Chen, J.J., et al. (2008). Inflammatory pain in the rabbit: a new, efficient method for measuring mechanical hyperalgesia in the hind paw. J. Neurosci. Methods168: 76-87.

Gediz, E.I., Nacitarhan, C., Minareci, E., and Sadan, G. (2015). Antinociceptive Effect of Vardenafil on Carrageenan-Induced Hyperalgesia in Rat: involvement of Nitric Oxide/Cyclic Guanosine Monophosphate/Calcium Channels Pathway. Iran. J. Pharm. Res. IJPR14 : 1137-1143.

Ghaisas, M.M., Dandawate, P.R., Zawar, S.A., Ahire, Y.S., and Gandhi, S.P. (2010). Antioxidant, antinociceptive and anti-inflammatory activities of atorvastatin and rosuvastatin in various experimental models. Inflammopharmacology $18:$ 169-177.

Gibson, M., Li, H., Coburn, J., Moroni, L., Nahas, Z., Bingham, C. 3rd, et al. (2014). Intra-articular delivery of glucosamine for treatment of experimental osteoarthritis created by a medial meniscectomy in a rat model. J. Orthop. Res. 32 : 302-309.

Henrotin, Y., Kurz, B., and Aigner, T. (2005). Oxygen and reactive oxygen species in cartilage degradation: friends or foes? Osteoarthr. Cartil. $13: 643-654$.

Hoeven, T.A., Kavousi, M., Clockaerts, S., Kerkhof, H.J.M., Meurs, J.B. van, Franco, O., et al. (2013). Association of atherosclerosis with presence and progression of osteoarthritis: the Rotterdam Study. Ann. Rheum. Dis. 72 : 646-651.

Hosseinzadeh, A., Bahrampour Juybari, K., Kamarul, T., and Sharifi, A.M. (2019). Protective effects of atorvastatin on high glucose-induced oxidative stress and mitochondrial apoptotic signaling pathways in cultured chondrocytes. J. Physiol. Biochem. $75:$ 153-162.

Hughes, A., Rogers, M.J., Idris, A.I., and Crockett, J.C. (2007). A comparison between the effects of hydrophobic and hydrophilic statins on osteoclast function in vitro and ovariectomy-induced bone loss in vivo. Calcif. Tissue Int. 81 : 403-413.

Jaiswal, S.R., and Sontakke, S.D. (2012). Experimental evaluation of analgesic and anti-inflammatory activity of simvastatin and atorvastatin. Indian J. Pharmacol. 44 : 475-479.

Janusz, M.J., Bendele, A.M., Brown, K.K., Taiwo, Y.O., Hsieh, L., and Heitmeyer, S.A. (2002). Induction of osteoarthritis in the rat by surgical tear of the meniscus: Inhibition of joint damage by a matrix metalloproteinase inhibitor. Osteoarthr. Cartil. 10 : 785-791.

Johnson, V.L., and Hunter, D.J. (2014). The epidemiology of osteoarthritis. Best Pract. Res. Clin. Rheumatol. 28: 5-15.

Kamel, E., Elsaid, A., Gumaa, E., and Sheweal, A.E. El (2016). Statins attenuate hyperalgesia and inflammation in experimentally induced acute and neuropathic pain in rats. Ain-Shams J. Anaesthesiol. 9 : 440. 
Kapoor, M., Martel-Pelletier, J., Lajeunesse, D., Pelletier, J.-P., and Fahmi, H. (2011). Role of proinflammatory cytokines in the pathophysiology of osteoarthritis. Nat. Rev. Rheumatol. $7: 33-42$.

Karasawa, K.Y. and R. (2010). Statin prevents chondrocyte aging and degeneration of articular cartilage in osteoarthritis ( OA ). Aging (Albany. NY). 2 : 990-998.

Katoh, A., Kai, H., Harada, H., Niiyama, H., and Ikeda, H. (2017). Oral Administration of Glucosamine Improves Vascular Endothelial Function by Modulating Intracellular Redox State. Int. Heart J. 58 : 926932.

Kavalipati, N., Shah, J., Ramakrishan, A., and Vasnawala, H. (2015). Pleiotropic effects of statins. Indian J. Endocrinol. Metab. 19:554-562.

Khan, H.M., Ashraf, M., Hashmi, A.S., Ahmad, M.U.D., and Anjum, A.A. (2013). Papain induced progressive degenerative changes in articular cartilage of rat femorotibial joint and its histopathological grading. J. Anim. Plant Sci. 23 : 350-358.

Kim, J.-W., Cho, H.-R., and Ku, S.-K. (2012). Efficacy test of Polycan, a beta-glucan originated from Aureobasidium pullulans SM-2001, on anterior cruciate ligament transection and partial medial meniscectomyinduced-osteoarthritis rats. J. Microbiol. Biotechnol.22 : 274-282.

Kuedo, Z., Sangsuriyawong, A., Klaypradit, W., Tipmanee, V., and Chonpathompikunlert, P. (2016). Effects of Astaxanthin from Litopenaeus Vannamei on Carrageenan-Induced Edema and Pain Behavior in Mice. Molecules $21: 382$.

Kuyinu, E.L., Narayanan, G., Nair, L.S., and Laurencin, C.T. (2016). Animal models of osteoarthritis: Classification, update, and measurement of outcomes. J. Orthop. Surg. Res. 11 : 1-27.

Maldonado, M., and Nam, J. (2013). The role of changes in extracellular matrix of cartilage in the presence of inflammation on the pathology of osteoarthritis. Biomed Res. Int. 2013 : 284873.

Marino-Martinez, I.A., Martinez-Castro, A.G., Pena-Martinez, V.M., Acosta-Olivo, C.A., Vilchez-Cavazos, F., Guzman-Lopez, A., et al. (2019). Human amniotic membrane intra-articular injection prevents cartilage damage in an osteoarthritis model. Exp. Ther. Med. 17: 11-16.

Marius, M., Jabeen, A., Gilbert, A., Simjee, S.U., Desire, B.T.F., and Dastagir, N. (2018). NO-cGMP-K channel-dependent anti-nociceptive activities of methanol stem bark extract of Piptadeniastrum africanum (Mimosaceae) on rats. Asian Pac. J. Trop. Biomed. 8 : 150-159.

Mobasheri, A., and Batt, M. (2016). An update on the pathophysiology of osteoarthritis. Ann. Phys. Rehabil. Med. 59 : 333-339.

Okhuarobo, A., and Ozolua, R. (2017). Analgesic and anti-inflammatory effects of the aqueous leaf extract of Dichrostachys cinerea.

Pathak, N.N., Balaganur, V., Lingaraju, M.C., Kant, V., Kumar, D., Kumar, D., et al. (2015a). Effect of atorvastatin, a HMG-CoA reductase inhibitor in monosodium iodoacetate-induced osteoarthritic pain: implication for osteoarthritis therapy. Pharmacol. Rep. 67: 513-519.

Pathak, N.N., Lingaraju, M.C., Balaganur, V., Kant, V., More, A.S., Kumar, D., et al. (2015b). Antiinflammatory and chondroprotective effects of atorvastatin in a cartilage explant model of osteoarthritis. Inflamm. Res. 64 : 161-169.

Phan, D.Q., Silka, M.J., Lan, Y.-T., and Chang, R.-K.R. (2015). Comparison of formulas for calculation of the corrected QT interval in infants and young children. J. Pediatr. 166 : 960-962.

Regan, E.A., Bowler, R.P., and Crapo, J.D. (2008). Joint fluid antioxidants are decreased in osteoarthritic joints compared to joints with macroscopically intact cartilage and subacute injury. Osteoarthr. Cartil. 16 : 515-521. 
Rezende, M.U. de, Gurgel, H.M. de C., Vilaca Junior, P.R., Kuroba, R.K., Lopes, A.S.S., Phillipi, R.Z., et al. (2006). Diacerhein versus glucosamine in a rat model of osteoarthritis. Clinics (Sao Paulo).61 : 461-466.

Rovati, L.C., Girolami, F., and Persiani, S. (2012). Crystalline glucosamine sulfate in the management of knee osteoarthritis: efficacy, safety, and pharmacokinetic properties. Ther. Adv. Musculoskelet. Dis.4 : 167-180.

Sadeghi, H., Hajhashemi, V., Minaiyan, M., Movahedian, A., and Talebi, A. (2013). Further studies on antiinflammatory activity of maprotiline in carrageenan-induced paw edema in rat. Int. Immunopharmacol.15 : 505-510.

Sadeghi, H., Mostafazadeh, M., Sadeghi, H., Naderian, M., Barmak, M.J., Talebianpoor, M.S., et al. (2014). In vivo anti-inflammatory properties of aerial parts of Nasturtium officinale. Pharm. Biol. 52 : 169-174.

Santodomingo-Garzon, T., Cunha, T.M., Verri, W.A.J., Valerio, D.A.R., Parada, C.A., Poole, S., et al. (2006). Atorvastatin inhibits inflammatory hypernociception. Br. J. Pharmacol. 149: 14-22.

Schmitz, N., Laverty, S., Kraus, V.B., and Aigner, T. (2010). Basic methods in histopathology of joint tissues. Osteoarthr. Cartil. 18 Suppl 3 : S113-6.

Setnikar, I., Cereda, R., Pacini, M.A., and Revel, L. (1991). Antireactive properties of glucosamine sulfate. Arzneimittelforschung.41: 157-161.

Shahine, E.M., and Elhadidi, A.S. (2014). Efficacy of glucosamine sulfate in lowering serum level of interleukin- $1 \beta$ in symptomatic primary knee osteoarthritis: Clinical and laboratory study. Alexandria J. Med.50 : 159-163.

Shiomi, T., Lemaitre, V., D’Armiento, J., and Okada, Y. (2010). Matrix metalloproteinases, a disintegrin and metalloproteinases, and a disintegrin and metalloproteinases with thrombospondin motifs in non-neoplastic diseases. Pathol. Int. 60 : 477-496.

Simopoulou, T., Malizos, K.N., Poultsides, L., and Tsezou, A. (2010). Protective effect of atorvastatin in cultured osteoarthritic chondrocytes. J. Orthop. Res. $28: 110-115$.

Singh, S., Khajuria, A., Taneja, S.C., Khajuria, R.K., Singh, J., and Qazi, G.N. (2007). Boswellic acids and glucosamine show synergistic effect in preclinical anti-inflammatory study in rats. Bioorg. Med. Chem. Lett. $17: 3706-3711$.

Solanki, H.K., Shah, D.A., Maheriya, P.M., and Patel, C.A. (2015). Evaluation of anti-inflammatory activity of probiotic on carrageenan-induced paw edema in Wistar rats. Int. J. Biol. Macromol. 72 : 1277-1282.

Tsezou, A., Iliopoulos, D., Malizos, K.N., and Simopoulou, T. (2010). Impaired expression of genes regulating cholesterol efflux in human osteoarthritic chondrocytes. J. Orthop. Res. 28 : 1033-1039.

Uzkeser, H., Cadirci, E., Halici, Z., Odabasoglu, F., Polat, B., Yuksel, T.N., et al. (2012). Anti-inflammatory and antinociceptive effects of salbutamol on acute and chronic models of inflammation in rats: involvement of an antioxidant mechanism. Mediators Inflamm. 2012 : 438912.

Waly, N.E., Refaiy, A., and Aborehab, N.M. (2017). IL-10 and TGF-beta: Roles in chondroprotective effects of Glucosamine in experimental Osteoarthritis? Pathophysiol. Off. J. Int. Soc. Pathophysiol. 24 : 45-49.

Wei, Y., Jia, J., Jin, X., Tong, W., and Tian, H. (2018). Resveratrol ameliorates inflammatory damage and protects against osteoarthritis in a rat model of osteoarthritis. Mol. Med. Rep. 17 : 1493-1498.

Wen, Z.-H., Tang, C.-C., Chang, Y.-C., Huang, S.-Y., Hsieh, S.-P., Lee, C.-H., et al. (2010). Glucosamine sulfate reduces experimental osteoarthritis and nociception in rats: association with changes of mitogenactivated protein kinase in chondrocytes. Osteoarthr. Cartil.18 : 1192-1202.

Winter, C.A., Risley, E.A., and Nuss, G.W. (1962). Carrageenin-induced edema in hind paw of the rat as an assay for antiiflammatory drugs. Proc. Soc. Exp. Biol. Med. 111 : 544-547. 
Table (1): maximum angle of extension, histopathological score, and biochemical changes in different OA groups.

\begin{tabular}{llll}
\hline Animal groups & Maximum angle of knee extension (degree) & Histopathological score & $\Sigma$ \\
\hline Control non-osteoarthritic group & $22(20-25)$ & $0 \pm 0$ & 0 \\
Non-treated OA group & $55(50-60) \#$ & $10.97 \pm 1.13^{\#}$ & 0 \\
Glucosamine treated OA group & $31(26-34) \# * \$$ & $3.9 \pm 0.52^{* *}$ & 0 \\
Atorvastatin treated OA group & $29(26-32) \#^{* \$}$ & $1.66 \pm 0.57^{* \$}$ & 0 \\
\hline
\end{tabular}

Data are presented as means $\pm \mathrm{SD}, \mathrm{n}=8$ rats in all except the maximum angle of knee extension presented as median (range).

Significant at $(\mathrm{p}<0.05)$.

\# Significance versus control normal group.

* Significance versus osteoarthritic group.

$\$$ Significance versus glucosamine treated group.

Figure legends

Figure (1) Maximum angle of knee extension with zero degree represent as the maximum possible extension (Rezende et al., 2006)

Figure (2) : The histopathological findings of different groups. I : Non-osteoarthritic group showing articular cartilage (AC) with its smooth surface (arrow). The chondrocytes appear in the matrix (curved arrow). Subchondral bone (B) and bone marrow (BM) are seen, allocated Grade-0. IIa : OA group showing marked erosion of articular cartilage surface with marked matrix loss (arrow), decreased number and size of chondrocytes with some cell death (curved arrow) and some cavities in the subchondral bone (asterisk), allocated Grade-4. IIb : OA group showing irregular surface (arrow), disorientation of chondrocytes with some cell death (arrowhead) and presence of multiple cavities (asterisk), allocated grade 3.III : Glucosamine treated OA group: Showing discontinuous superficial layer of articular cartilage (arrow), increase in the number of chondrocytes, with cluster formation (arrowhead), some cell death (asterisk), matrix loss (tailed arrow) and increase in cartilage thickness, allocated Grade-2 (Group 3, H \& E X 400). IV : diacerein treated OA group showing an irregular surface of articular cartilage (arrow), increase in the number of chondrocytes, disturbed morphology with cluster formation (arrowhead), some cell death (asterisk) and increase the cartilage thickness, allocated Grade-2.V : atorvastatin treated OA group showing a nearly smooth surface of articular cartilage (arrow), a slight increase in the number of chondrocytes with the initiation of cluster formation, condensation of cells in the superficial layer (arrowhead) and increase the cartilage thickness, allocated Grade-1. (H\&E, X400).

Figure (3): Effect of different drugs on paw thickness using carrageenan induced paw edema test.

Data are presented as means \pm SD.

Significant at $(\mathrm{p}<0.05)$.

\# Significance versus control normal group.

* Significance versus Carrageenan treated group.

Significance versus indomethacin +

pretreated group. 
\$ Significance versus glucosamine pretreated group.

Figure 4: Effect of atorvastatin, diacerein or glucosamine sulfate on nociceptive threshold using carrageenan induced hyperalgesia test.

Data are presented as means \pm SD.

Significant $(\mathrm{p}<0.05)$.

\# Significance versus control normal group.

* Significance versus Carrageenan treated group.

Significance versus indomethacin treated group.

\$ Significance versus glucosamine treated group.
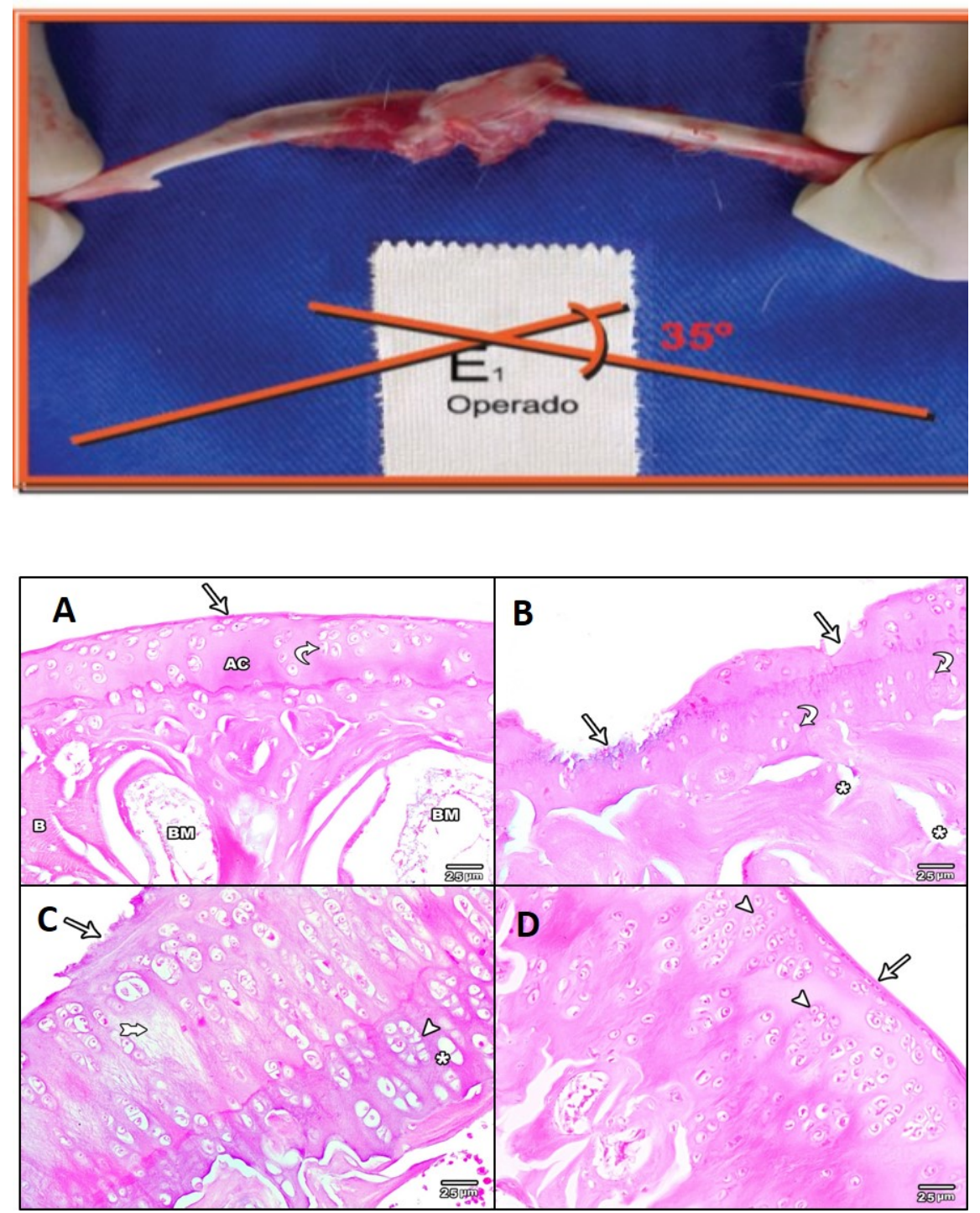

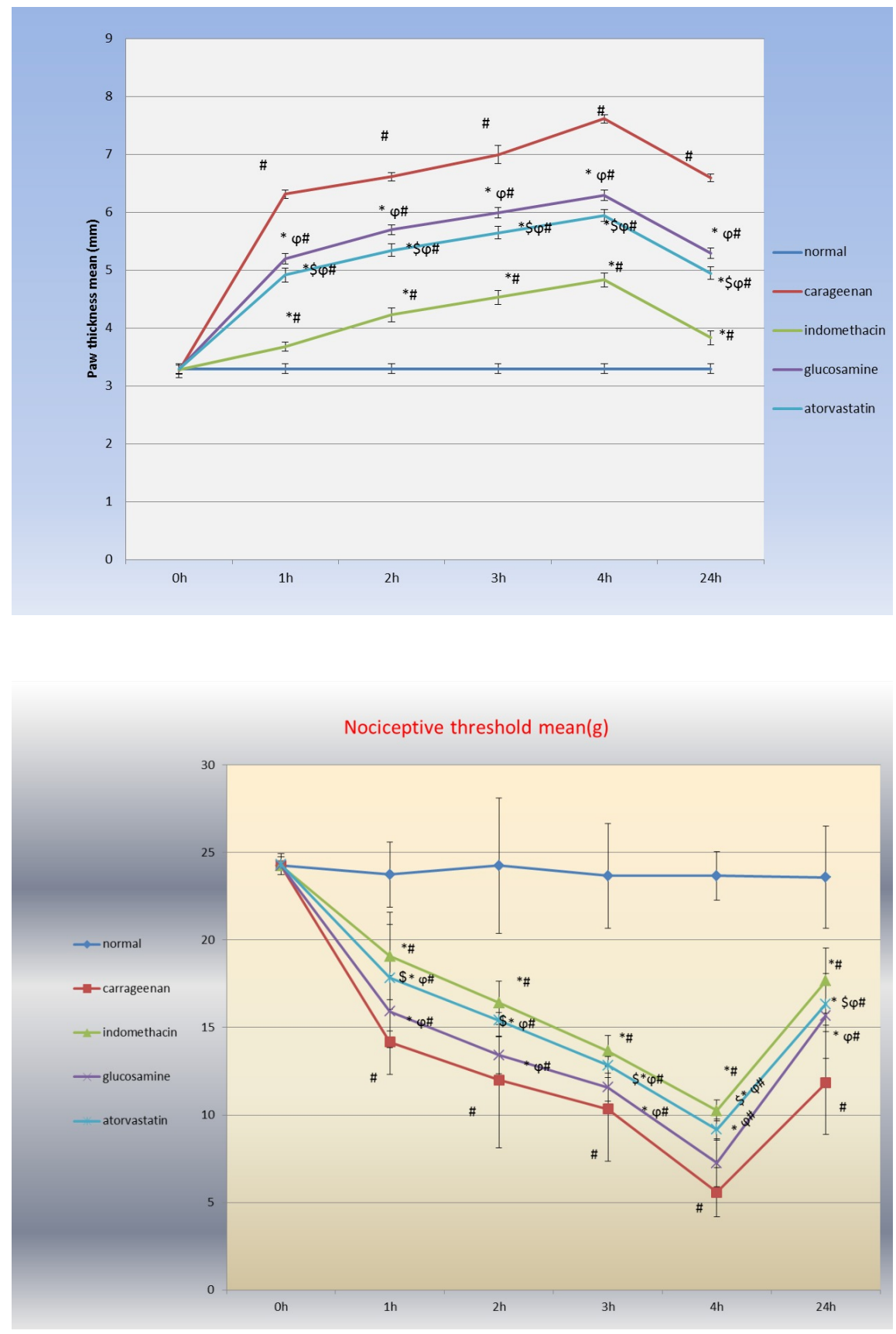

\section{Hosted file}

Table.pdf available at https://authorea.com/users/375388/articles/492637-standardized-studyof-atorvastatin-possible-osteoarthritis-disease-modifying-effect-in-rats 